to the HLA typing results. HLA typing results were also compared to the tag SNP rs1061235 results to calculate the false positive rate. Results. There was $100 \%$ concordance between our real-time PCR results and expected results based on HLA typing. 89 sample results for tag SNP rs1061235 were compared to HLA typing results. 75/89 samples had a rs 1061235 variant, but $31 / 75(41 \%)$ samples did not have the HLA-A*31:01 type, thus defining the false positive rate of the tag SNP for our population. We theorized there would be a small subset of rare HLA-A types that would interfere with the assay and we tested the three types available to us. We confirmed that 3 of the HLA types (HLA-A*31:04, 31:12, and 31:16) result falsely positive due to sequence homology with $31: 01$. There is no known literature indicating whether these rare HLA-A*31 subtypes are associated with cutaneous adverse reactions. These 3 HLA types and the other suspected interfering HLA types have limited frequency data sets and are expected to occur rarely in our patient population; we expect these HLA types make up less than $0.003 \%$ of the our population. Our assay specificity for the validation is $>99 \%$. Conclusions. Our custom real-time PCR assay for detection of HLA-A ${ }^{\star} 31: 01$ is significantly more specific than the commonly used tag SNP rs1061235. Clinicians considering carbamazepine therapy for their patients will have a better understanding of cutaneous adverse reaction risk and can make improved personalized treatment decisions. This quick, cost effective assay allows more patients in need of carbamazepine treatment to benefit from its use. Funding. Genomind, Inc.

\section{Can Low Dose Sertraline Cause Serotonin Syndrome in Pediatric Patients? 2 Case Reports}

Sultana Jahan, MD

University of Missouri, Department of Psychiatry, Columbia, MO, USA

Presenting Author: Sultana Jahan

\begin{abstract}
Background. Serotonin syndrome is a potentially life-threatening condition associated with increased serotonergic activity in the central nervous system. Serotonin syndrome is underreported complication of pharmacotherapy. The Hunter Criteria for serotonin syndrome (SS) are fulfilled if the patient has taken a serotonergic agent and has one of the following symptoms: 1) spontaneous clonus, 2) inducible clonus and agitation or diaphoresis, 3) ocular clonus and agitation or diaphoresis, 4) tremor and hyperreflexia, 5) hypertonia, or 6) temperature above $38 \mathrm{C}$ and ocular clonus or inducible clonus.

Method. Patient A was a 16-year-old Caucasian male with history of major depressive disorder, social anxiety and OCD who presented to the emergency room with multiple complaints: twitching of bilateral cheeks, intermittent tremor of his hands and feet, mental fogginess/confusion, stuttering when attempting to speak, agitation, profuse sweating and headache. 3 weeks prior, his sertraline dose was increased from $25 \mathrm{mg}$ daily to $50 \mathrm{mg}$ daily. His physical exam was remarkable for elevated blood pressure and
\end{abstract}

heart rate as well as hyperreflexia noted on patellar reflex testing. No significant abnormalities were noted on routine labs. He was told his symptoms were likely due to medication side effects. The patient was discharged with instructions to decrease his sertraline dose from $50 \mathrm{mg}$ to $25 \mathrm{mg}$ daily and follow up with his outpatient psychiatrist. 2 days later the patient was seen at the outpatient child psychiatry clinic and he was advised to taper off sertraline completely by taking $12.5 \mathrm{mg}$ daily for 3 days before cessation. After stopping the medication, the patient's symptoms resolved. Patient B was a 16-year-old female with generalized anxiety disorder and major depressive disorder who presented to the general pediatric clinic with progressively worsening hand tremors and body shaking since her Zoloft dose was increased from $25 \mathrm{mg}$ to $50 \mathrm{mg}$ daily. She also felt it was more difficult to hold objects. At the physical exam she had an elevated heart rate to 93 and elevated blood pressure to $182 / 75$. Her deep tendon reflexes were $4+$ bilaterally. Upon consultation with child psychiatry, the patient was recommended to taper off sertraline. After the discontinuation of sertraline, her symptoms resolved.

Result. These 2 patients developed mild to moderate symptoms of serotonin syndrome with low doses of sertraline. Symptoms resolved after the discontinuation of the SSRI.

Discussion. In the pediatric patient population, serotonin syndrome can develop even with lower doses of an SSRI. To avoid a missed diagnosis, clinicians should familiarize themselves with the Hunter Criteria for serotonin syndrome. It is also vital to educate parents and caregivers about the toxicities of SSRIs, including serotonin syndrome, so they may monitor treatment and take appropriate action if needed.

\section{Phase 3 Safety and Tolerability Results of the Combination Olanzapine and Samidorphan in Patients with Schizophrenia: The 1 Year ENLIGHTEN-2-Extension}

\author{
Rene Kahn, $\mathrm{MD}, \mathrm{PhD}^{1}$, Bernard Silverman, $\mathrm{MD}^{2}$, \\ Lauren DiPetrillo, $\mathrm{PhD}^{2}$, Christine Graham, $\mathrm{PhD}^{2}$, \\ Ying Jiang, $\mathrm{PhD}^{2}$, Jiani Yin, $\mathrm{PhD}^{2}$, Adam Simmons, $\mathrm{MPH}^{2}$, \\ Vasudev Bhupathi, $\mathrm{MS}^{2}$, Bei $\mathrm{Yu}, \mathrm{MD}, \mathrm{PhD}^{2}$, \\ Craig Hopkinson, $M^{2}$ and Davidd McDonnell, $M^{3}$
}

${ }^{1}$ Icahn School of Medicine at Mount Sinai, New York, NY, USA, ${ }^{2}$ Alkermes, Inc., Waltham, MA, USA, and ${ }^{3}$ Alkermes Pharma Ireland Limited, Dublin, Ireland

\section{Presenting Author: Adam Simmons}

\section{Abstract}

Objective. Combination olanzapine and samidorphan (OLZ/SAM) is in development for treatment of schizophrenia and bipolar I disorder and is intended to provide the antipsychotic efficacy of olanzapine while mitigating olanzapine-associated weight gain. This 52-week open-label extension study (NCT02873208; ENLIGHTEN2-EXT) in schizophrenia assessed the safety and tolerability of OLZ/SAM. Methods: Patients completing the 24-week, randomized, 
double-blind, phase 3 ENLIGHTEN-2 study comparing weight gain with OLZ/SAM vs olanzapine were eligible for ENLIGHTEN-2-EXT enrollment. Initial OLZ/SAM doses were based on olanzapine dose (10 or $20 \mathrm{mg}$ ) received at the conclusion of ENLIGHTEN-2; subsequent olanzapine dose adjustments were allowed. The samidorphan dose $(10 \mathrm{mg})$ remained fixed throughout. Assessments included adverse events (AEs), weight, waist circumference, metabolic laboratory parameters, and Positive and Negative Syndrome Scale (PANSS) scores. Analyses were based on observed results using descriptive statistics. Baseline was relative to the first OLZ/SAM dose in the extension study.

Results. 265 patients received OLZ/SAM; 167 (63.0\%) completed the extension study. Common AEs $(=5 \%)$ were weight decreased $(n=23 ; 8.7 \%)$, extra dose administered $(n=21 ; 7.9 \%)$, headache $(\mathrm{n}=18 ; 6.8 \%)$, and weight increased $(\mathrm{n}=16 ; 6.0 \%)$. At week 52 , mean (SD) change from baseline for weight and waist circumference was $-0.03(6.216) \mathrm{kg}$ and $-0.35(6.115) \mathrm{cm}$, respectively. Changes in fasting lipid and glycemic parameters were generally small and remained stable over 52 weeks. PANSS total scores remained stable during the extension.

Conclusions. OLZ/SAM was generally well tolerated over 52 weeks. Weight, waist circumference, metabolic laboratory parameters, and schizophrenia symptoms remained stable throughout the study.

Funding. Alkermes, Inc.

\section{Deutetrabenazine Reduces Severe Tardive Dyskinesia Movements in a 3-year Open- Label Extension Trial}

\author{
Nayla Chaijale, $\mathrm{PhD}^{1}$, Joseph Bona, $\mathrm{MD}^{2 \dagger}$, \\ Hadas Barkay, $\mathrm{PhD}^{3}$, Amanda Wilhelm, $\mathrm{PhD}^{1}$ and \\ Mark Forrest Gordon, MD ${ }^{1}$
}

${ }^{1}$ Teva Pharmaceutical Industries Ltd., West Chester, PA, USA, ${ }^{2}$ Emory University, Atlanta, GA, USA, and ${ }^{3}$ Teva Pharmaceutical Industries Ltd., Netanya, Israel

Presenting Author: Nayla Chaijale
Results. 337 patients were analyzed. The upper quartile of baseline total AIMS score was 14. Subgroups were defined as $>14$ and $\leq 14$ at baseline, respectively ( $\mathrm{n}=64$ vs 273 ); data are presented at Week $145(n=40$ vs 120$)$. Mean treatment duration was 880.5 and 760.8 days. Mean \pm SE daily doses were $41.1 \pm 1.6 \mathrm{mg}$ and $38.9 \pm 1.0 \mathrm{mg}$. Mean $\pm \mathrm{SE}$ change from baseline in AIMS score was $-11.0 \pm 0.8$ versus $-5.1 \pm 0.3$; percent change from baseline was $-60.1 \% \pm 3.6 \%$ versus $-55.9 \% \pm 3.0 \%$. More patients with AIMS score $>14$ had $\geq 50 \%$ AIMS reduction ( $73 \%$ vs $65 \%$ ). Less patients discontinued ( $38 \%$ vs $51 \%$ ); reasons included withdrawal by subject ( $16 \%$ vs $25 \%$ ), adverse event ( $3 \%$ vs $11 \%$ ), and lost to follow-up (6\% vs $7 \%)$. Withdrawal due to lack of efficacy was uncommon ( $5 \%$ vs $2 \%)$.

Conclusions. Patients with baseline total AIMS score $>14$ had clinically meaningful reductions in AIMS score, suggesting deutetrabenazine has long-term benefit in these patients.

Funding. Teva Pharmaceutical Industries Ltd., Petach Tikva, Israel

${ }^{\dagger}$ Dr. Bona died on June 1, 2020. Prior to his passing, Dr. Bona was instrumental in designing the post-hoc analyses described in this abstract and interpreted the interim data results for these analyses; however, he was not able to finalize this abstract.

\section{Qualitative Clinical Trial Exit Interviews Evaluating Treatment Benefit, Burden, and Satisfaction in Patients with Schizophrenia}

\author{
Adam Simmons, $\mathrm{MPH}^{1}$, Julia Carpenter-Conlin, $\mathrm{MSW}^{1}$, \\ Leona Bessonova, $\mathrm{PhD}^{1}$, Amy K. O'Sullivan, $\mathrm{PhD}^{1}$, \\ David McDonnell, $\mathrm{MD}^{2}$, Cory Saucier, $\mathrm{MPH}^{3}$, \\ Michelle K. White, $\mathrm{PhD}^{3}$, April M. Foster, $\mathrm{BS}^{3}$, \\ Jakob B. Bjorner, MD, $\mathrm{PhD}^{3}$, Olga Lapeyra, MD, CCRP ${ }^{4}$ and \\ David P. Walling, PhD $^{5}$
}

\footnotetext{
${ }^{1}$ Alkermes, Inc., Waltham, MA, USA, ${ }^{2}$ Alkermes Pharma Ireland Limited, Dublin, Ireland, ${ }^{3}$ Optum, Inc., Johnston, RI, USA, ${ }^{4}$ Segal Trials, Miami, FL, USA, and ${ }^{5} \mathrm{CNS}$ Network, LLC, Garden Grove, CA, USA
}

\begin{abstract}
Background. There are no established treatment guidelines for tardive dyskinesia (TD) based on movement severity. The 12-week ARM-TD and AIM-TD studies in TD patients with baseline Abnormal Involuntary Movement Scale (AIMS) total score (items $1-7$ ) $\geq 6$ showed clinically significant improvements in AIMS score with deutetrabenazine versus placebo. Patients who completed these studies were eligible for the open-label extension (OLE) trial. This post-hoc analysis evaluated deutetrabenazine in TD patients with severe movements.

Methods. Subgroups were defined by upper quartile of baseline total AIMS score (local rating). Endpoints were: change and percent change from baseline in AIMS score, and percent of patients achieving $\geq 50 \%$ AIMS reduction from baseline.
\end{abstract}

Presenting Author: Adam Simmons
Abstract
Objective. An open-label extension study (NCT02873208) eval- uated the long-term tolerability, safety, and efficacy of combina- tion olanzapine/samidorphan (OLZ/SAM) treatment in patients with schizophrenia. This qualitative sub study explored percep- tions of benefit, burden, and satisfaction with previous medica- tions and OLZ/SAM.
Methods. Semi-structured interviews (60 minutes; audio- recorded) were conducted. Interviewer sensitivity training, senior interviewer oversight, and a list of common medications to aid recall supported data collection. Interview transcripts were con- tent coded and analyzed (NVivo v11.0). 